\title{
Fundamentos de la Auditoría Ambiental con una visión de Responsabilidad Social
}

\section{Basics of environmental auditoring with social responsibility perspective}

DOI: $10.46932 / s f j d v 2 n 3-043$

Received in: May 1st, 2021

Accepted in: Jun 30th, 2021

\author{
Ing. Verónica Ochoa Hidalgo \\ MAE \\ Universidad Laica VICENTE ROCAFUERTE de Guayaquil (ULVR) \\ vochoah@ulvr.edu.ec \\ Universidad Internacional del Ecuador (UIDE) \\ E-mail: veochoahi@uide.edu.ec \\ PhD. Washington Villavicencio Santillán \\ $\mathrm{Mg}$. MSc \\ Universidad Laica VICENTE ROCAFUERTE de Guayaquil (ULVR) \\ E-mail: wvillavicencios@ulvr.edu.ec \\ Ec. Cindy Loor Mero \\ MSc \\ Universidad Internacional del Ecuador (UIDE) \\ E-mail: ciloorme@uide.edu.ec
}

\section{RESUMEN}

La Auditoría Ambiental en la actualidad es necesaria como un indicador que evalúa los esfuerzos que las organizaciones realizan por alcanzar la protección del medio ambiente, la misma que debe ser confiable y adecuada, todo esto se realiza en el marco de que las empresas deben de trabajar con una perspectiva de responsabilidad social ambiental, que permita el crecimiento y la sostenibilidad de la organización enmarcados en la normativa internacional ambiental como es la ISO 14001:2009, en donde se establecen políticas y objetivos que las organizaciones deben de seguir y cumplir con la finalidad de obtener metodologías adecuadas de sistemas de gestión ambiental que ayuden a optimizar y mejorar sus procesos y disminuir el impacto en el ambiente. El compromiso de los docentes que realizamos esta investigación de auditoría ambiental es el de socializar y estandarizar en las organizaciones hoteleras lo necesario que es proteger el medio ambiente, la salud de los que en el viven y de lograr mantener un nivel de responsabilidad ambiental y a la vez la adaptación de las normas internacionales les ayude al ahorro de costos en los procesos que realizan manejando sistemas integrados, teniendo un sentido de ética y responsabilidad social y moral en los cambios que se tienen que dar para que poder construir un ecosistema más saludable y que nos permita innovar y competir con organizaciones hoteleras de países vecinos que si manejan estándares de calidad en sus procesos.

Palabras Claves: Auditoría Ambiental, Normas ISO, Responsabilidad Social, Sector Hotelero

\section{ABSTRACT}

Environmental audit is needed today as an indicator that assesses the efforts made by organizations achieve environmental protection, it should be reliable and adequate, all this is done within companies should work with a perspective of environmental social responsibility, which allows the growth and sustainability of the organization framed in international environmental legislation such as the ISO 14001: 
2009, where established policies and objectives that organizations must follow and enforce order to obtain adequate methodologies environmental management systems that help optimize and improve their processes and reduce the impact on the environment. The commitment of teachers who conducted this research environmental audit is to socialize and the hotel organizations to standardize on how necessary it is to protect the environment, the health of those who live in and manage to maintain a level of environmental responsibility and After the adaptation of international standards will help them to save costs in managing the processes performed integrated systems, having a sense of ethics and social and moral responsibility for the changes that need to take to be able to build a healthier ecosystem and allow us to innovate and compete with hotel organizations from neighboring countries if they manage quality standards in its processes.

Key words: Environmental audit, ISO Standards, Social Responsibility, Hotel Sector

\section{INTRODUCCIÓN}

La Responsabilidad Social (RS) ha sido un tema recurrente en los últimos años debido a su importancia por presentar un enfoque integrador en los diversos campos de actuación lo que genera cambios que apuntan a la preservación y conservación del medio ambiente lo cual consolida el crecimiento y la sostenibilidad del progreso del país.

El Ecuador es reconocido a nivel mundial por su riqueza en flora y fauna, además de contar con el Parque Nacional Yasuni ITT que forma parte de la Reserva Mundial de Biosfera dentro del programa de la UNESCO.

El propósito del presente estudio es relevar la importancia de la Auditoria Ambiental, para estandarizar las políticas públicas y privadas y así contribuir al desarrollo sostenible del sector empresarial de la ciudad de Guayaquil con esto aportar a la Matriz Productiva y al Plan Nacional del Buen Vivir.

\subsection{AUDITORÍA AMBIENTAL}

La auditoría ambiental (AA) es un excelente recurso de planificación y gestión empresarial que permite enfrentar con éxito los requerimientos del tratamiento del medio ambiente (Cuevas Barros, C., \& Cuevas Casas, C, 2013) Por el contrario otro autor explica que la auditoría ambiental es un "Examen analítico de las operaciones de una organización relacionadas con la contaminación y el riesgo ambiental que conllevan, grado de cumplimiento de la legislación medio ambiental y de los parámetros internacionales, con el objeto de mejorar la eficiencia y eficacia de sus procesos en su desempeño ambiental e implementar medidas preventivas y correctivas para proteger el medioambiente". (Franklin, 2015) 
La Auditoría Ambiental es un examen metodológico de los procesos de una empresa de carácter técnico que tiene por objetivo supervisar el cumplimiento de las normas de protección al medio ambiente en obras y trabajos de desarrollo en el manejo sustentable de los recursos naturales.

La AA (Auditoria Ambiental) son aquellas que te permiten evaluar la eficiencia y eficacia del sistema de gestión ambiental en las empresas. Este sistema se estima mediante las normas ISO 140012015 o el reglamento EMAS. Esta auditoria ambientales les proporcionas confianzas a las empresas u organizaciones sobre el sistema de gestión ambiental, si se da el cumplimiento de las normas y visualizar los puntos débiles y realizar las mejoras.

Sin embargo, para el autor (Sotomayor, 2008), por medio de la auditoría ambiental se realizan exámenes técnicos relacionados con el impacto industrial y de desechos sobre el medio ambiente y los recursos naturales, situación que se agrava día tras día y requiere la implementación de medidas preventivas.

La finalidad de realizar una AA (Auditoria Ambiental) es salvaguardar el medio ambiente y los recursos naturales utilizados en los proyectos, hacer conciencia con la preservación del ecosistema entre los empleados, los clientes, público en general y los directivos mediante las leyes ambientales nacionales e internacionales.

Sin embargo, (Mejía, 2015),considera que se debe incluir tres dimensiones: La economía, la social y la ambiental.

La gestión ambiental esta enlazada con el desarrollo industrial ambos deben estar equilibrados y es esencial para la generación de recursos, lo que permite que se generen empleos y que se impulsen la educación ambiental como fundamento en el bienestar social y mejorar la calidad de vida y asi lograr el desarrollo sostenible.

(ATRISTAIN - ÁLVAREZ, 1999)“La auditoría es el examen realizado por el auditor que proporciona evidencias para formular y emitir un informe donde plasmará su opinión sobre los elementos revisados, este informe representa la conclusión del trabajo de auditoria, para salvaguardar el medio ambiente, la auditoría ambiental contribuye a facilitar la administración, el control de las prácticas ambiéntales y verificar el cumplimiento de las políticas de la compañía, las cuales pueden incluir requerimientos de regulaciones". (Pág. 117)

La auditoría ambiental es un método de control sistemático, periódico, documentado, y objetivo que permite tener información de la organización con relación a su gestión ambiental, para verificar en qué medida se ajusta al cumplimiento de criterios de auditorías específicas.

(PEQUERO - MEDINA - CAMPOS, 2008) La auditoría ambiental consiste en la evaluación de la organización del sistema de gestión y equipo con que cuenta la planta o centro de trabajo, realizando una 
comparación entre las normas legales y las establecidas por la administración de la entidad, y los óptimos posibles, las normas legales y las establecidas por la administración de la entidad, y los óptimos posibles (tecnología de punta, la mejor tecnología de punta(tecnología de punta, la mejor tecnología disponible, etc.,) tratando de armonizar las normas internas, el proceso productivo particular de la entidad y las disposiciones legales. (Pág. 68)

La auditoría ambiental es una fotografía instantánea de la situación ambiental en una organización; la que no pretende predecir los impactos potenciales de inversiones planificadas. Sin embargo las auditorías ambientales se utilizan para evaluar la implementación de proyectos nuevos en donde se solicitan requisitos derivados de una evaluación ambiental.

\subsection{OBJETIVOS DE LA AUDITORIA AMBIENTAL}

Según la Norma ISSO 7750 (citado por Sánchez; 1998), cabe a la auditoria verificar:

Si las actividades de gestión ambiental están conforme el manual, programa, procedimiento e instrucciones de trabajo para la gestión ambiental, y si son implementadas efectivamente.

La eficacia del sistema de gestión ambiental para alcanzar los objetivos de la política ambiental de la organización

De acuerdo con Sánchez (1998), las funciones que debe cumplir la auditoría ambiental son:

- Verificar el cumplimiento de normas y patrones ambientales.

- Verificar el cumplimiento de objetivos de política empresarial.

- Identificar las fallas de funcionamiento y evaluar la eficiencia del proceso productivo

- Verificar la capacidad de la empresa para identificar en caso de accidente ambiental

- Minimizar el riesgo de acciones juridiciales con el objetivo de la reparación de daños ambientales.

- Mejorar la relación de la empresa con la comunidad y los órganos gubernamentales.

- Aumentar la conciencia de los problemas ambientales en trabajadores y gerentes.

- Verificar la precisión de las previsiones de impacto hechas en oportunidad de la planificación del emprendimiento.

- Facilitar el análisis de desempeño ambiental y proporcional elementos para la preparación de informes de desempeño ambiental y de otros medios de comunicación con las partes interesadas.

- Subsidiar la evaluación del pasivo ambiental de una empresa o de un inmueble.

De acuerdo con (ATRISTAIN - ÁLVAREZ, 1999 pág. 17), los beneficios derivados de la auditoría ambiental lo siguientes: 
- Facilita la comprensión e intercambio entre plantas o empresas.

- Incrementa el conocimiento de los empleados sobre políticas y responsabilidad ambientales.

- Identifica costos de salvamento potenciales, incluyendo aquellos derivados de la minimización de desperdicios.

- Facilitan la obtención de la certificación ISO 14000, pues es uno de los procedimientos requeridos dentro de la misma norma.

- Evalúa los programas de entrenamiento y proporciona datos para asesorar en el entrenamiento personal.

- Proporciona información para situaciones de emergencia y evalúa efectividad de respuesta de los sistemas de emergencia.

- Asegura una adecuada base de datos ambiental, actualizada, para el conocimiento y la toma de decisiones por parte de la administración de la empresa.

- Permite que la administración dé credibilidad a la información que presenta un buen funcionamiento de la empresa en cuestiones ambientales.

Según (Antúnez Sánchez, 2015), el interés de este ha alcanzado importancia y magnitud en los últimos años al convertirse en temática para el empresariado.

Son visibles los cambios que esta experimentado la tierra a consecuencia de la desforestación, la contaminación en el ambiente por la emisión de gases. Por eso son las iniciativas gubernamentales para enfrentar los cambios climáticos, mediante las auditorías ambientales las empresas pueden hacer conciencia y contribuir al cuidado del ecosistema y del entorno con el fin de mirar al futuro con mayores posibilidades y crecimientos sostenibles.

\subsection{TIPOS DE AUDITORÍAS AMBIENTALES}

(Reyes y Moreno, 2017) señalan que existen diversos tipos de AA, en función de las situaciones de la empresa. Las auditorías ambientales utilizadas con mayor frecuencia se presentan a continuación en la Tabla 1. 
Tabla 1. Tipos de auditorías ambientales

\begin{tabular}{|l|l|}
\hline TIPOS & DESCRIPCIÓN \\
\hline Auditoría ambiental externa & $\begin{array}{l}\text { Es ejecutada por auditores independientes, lo cual permiten una mejor } \\
\text { efectividad en los resultados. }\end{array}$ \\
\hline Auditoría ambiental interna & $\begin{array}{l}\text { Es realizada por auditores de la entidad, lo cual permite realizar } \\
\text { procedimientos de control ambiental interno con un menor presupuesto. }\end{array}$ \\
\hline $\begin{array}{l}\text { Auditoría Preliminar o de } \\
\text { Diagnostico }\end{array}$ & $\begin{array}{l}\text { Permite establecer un proyecto de mejoras medioambientales y un sistema } \\
\text { de gestión de recursos naturales, ya que reconoce los aspectos específicos } \\
\text { que afectan el medio ambiente. }\end{array}$ \\
\hline $\begin{array}{l}\text { Auditoría de Cumplimiento } \\
\text { Legal }\end{array}$ & $\begin{array}{l}\text { Se asegura que la entidad sea responsable con respecto al cumplimiento de } \\
\text { las leyes ambientales actuales ycuerdos legales que reduzcan la intensidad } \\
\text { de los daños al medioambiente. }\end{array}$ \\
\hline $\begin{array}{l}\text { Auditoría de un Sistema de } \\
\text { Gestión Ambiental }\end{array}$ & $\begin{array}{l}\text { Monitorea el proceso de gestión de recursos naturales existente en una } \\
\text { organización privada o del estado, inclusive la supervisión del cumplimiento } \\
\text { de los sistemas de gestión medioambiental, su importancia y la efectiva } \\
\text { administración. }\end{array}$ \\
\hline $\begin{array}{l}\text { Auditoría de Riesgos } \\
\text { Ambientales }\end{array}$ & $\begin{array}{l}\text { Reconoce los peligros que podrían surgir en las actividades y procesos de las } \\
\text { organizaciones. }\end{array}$ \\
\hline Auditorías de Residuos & $\begin{array}{l}\text { Identifican y cuantifican los distintos recorridos residuales, examinando así } \\
\text { los procesos para su gestión, estimando de esta forma los costos que } \\
\text { demandan. }\end{array}$ \\
\hline Auditoría de Procesos & $\begin{array}{l}\text { Se asegura de que los indicadores que miden los procesos de interés estén } \\
\text { siendo monitoreados. }\end{array}$ \\
\hline Auditoría Energética & $\begin{array}{l}\text { Estima la efectividad de la administración de los recursos energéticos de las } \\
\text { entidades. }\end{array}$ \\
\hline
\end{tabular}

Fuente: Elaboración propia a partir de Reyes y Moreno (2017).

\subsection{PRINCIPIOS GENERALES DE LA AUDITORIA AMBIENTAL}

Para poner en marcha una auditoría ambiental es imprescindible la responsabilidad de la firma auditora, la misma que debe mantener el compromiso en pos de las mejoras continuas de la práctica ambiental.

De acuerdo con (GABIÑO, 1999) la auditoría ambiental requiere de los siguientes principios:

○ Información suficiente y apropiada acerca de la operación, organización o sitio.

○ Disponibilidad de los recursos para apoyar el proceso de auditoría.

- Adecuada cooperación por parte de la compañía u otra entidad que está siendo auditada (auditado), $\mathrm{y}$

○ Un protocolo de auditoría (ejemplo, lista de chequeo o cuestionario).

De lo previamente expuesto surgen otras consideraciones de carácter particular que deben ser atendidas, como:

○ Una auditoría ambiental es iniciada por los auditores y está sustentada en los objetivos definidos por el cliente, el que también puede ser auditado. 
- Los criterios de auditoría son acordados entre los auditores y el cliente e informados al auditado con los objetivos y alcance de la auditoría.

○ Los auditores deben ser objetivos e independientes del sitio o actividad que está siendo auditada.

- La información recolectada en la auditoría siempre debe ser considerada como confidencial. (ISO 14001, 2015)

\subsection{NORMAS ISO}

La norma (ISO 14001, 2015)ha sido diseñada para poder ser implementada en cualquier organización, independientemente de su tamaño, sector y ubicación geográfica. Al momento que las empresas gestionan la certificación ISO 14001, pueden obtener algunos beneficios, entre ellos: reducir los costes, gestión del cumplimiento de la legislación, reducir la duplicación de esfuerzos, gestionar su reputación, entre otros.

Las auditorías ambientales se basan en las Normas Internacionales ISO, por sus siglas en inglés (Organización Internacional de Normalización). Las Normas Internacionales se redactan de acuerdo con las reglas establecidas en la Parte 2 de las Directivas ISO/CEI.

“La tarea principal de los comités técnicos es preparar Normas Internacionales. Los Proyectos de Normas Internacionales aceptados por los comités técnicos son enviados a los organismos miembros para votación. La publicación como Norma Internacional requiere la aprobación por al menos el 75 \% de los organismos miembros requeridos para votar. Se llama la atención sobre la posibilidad de que algunos de los elementos de esta Norma Internacional puedan estar sujetos a derechos de patente. ISO no asume la responsabilidad por la identificación de cualquiera o todos los derechos de patente.” (ISO 14001, 2015)

La auditoría ambiental la regula la Norma ISO 14001:2004, la misma que tiene como finalidad facilitar a las organizaciones los manuales de un Sistema de Gestión Ambiental (SGA) eficaz que puedan ser integrados con otros requisitos de gestión, y para ayudar a las organizaciones a lograr metas ambientales y económicas.

La Norma ISO 14001:2015 se basa en la metodología de Planificar, Hacer, Verificar y Actuar (PHVA), como se observa en la Figura 1. 
Figura 1. PHVA (Planificar, Hacer, Verificar, Actuar)

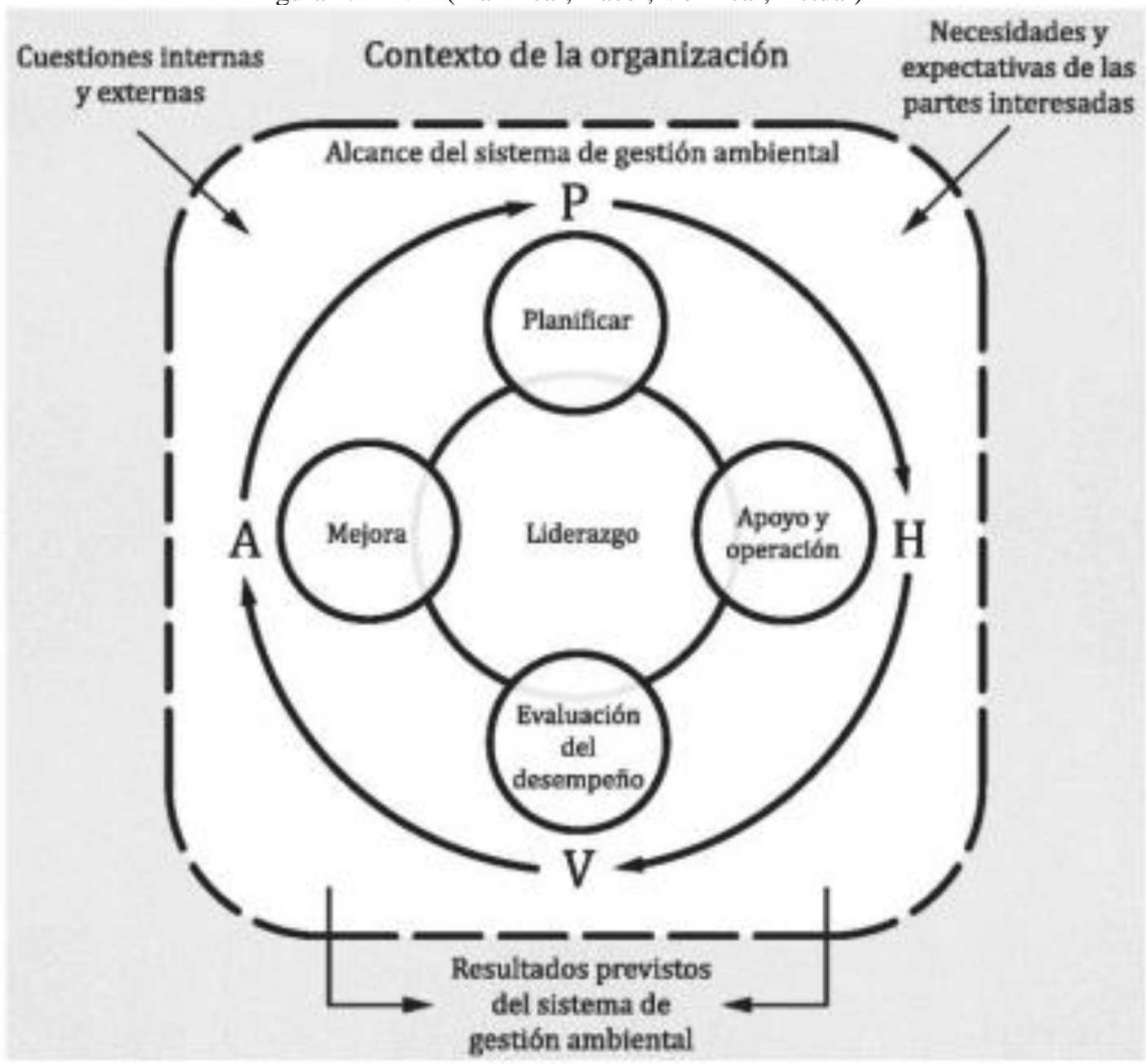

Fuente: (ISO 14001, 2015)

Los requisitos para un sistema de gestión ambiental según la Norma ISO 14001:2004 es permitir que las organizaciones desarrollen e implementen políticas y objetivos que tomen en cuenta los requisitos legales y otros requisitos que la organización suscriba, y la información relativa a los aspectos ambientales significativos. No implanta por sí misma criterios de desempeño ambiental específicos.

La Norma Internacional (ISO 14001, 2015) ayuda a una organización a lograr los resultados previstos de su sistema de gestión ambiental, con lo que aporta valor al medio ambiente, a la propia organización y a sus partes interesadas. En coherencia con la política ambiental de la organización, los resultados previstos de un sistema de gestión ambiental incluyen:

a) La mejora del desempeño ambiental

b) El cumplimiento de los requisitos legales y otros requisitos

c) El logro de los objetivos ambientales. 
Figura 2. Sistema Integrado de Gestión

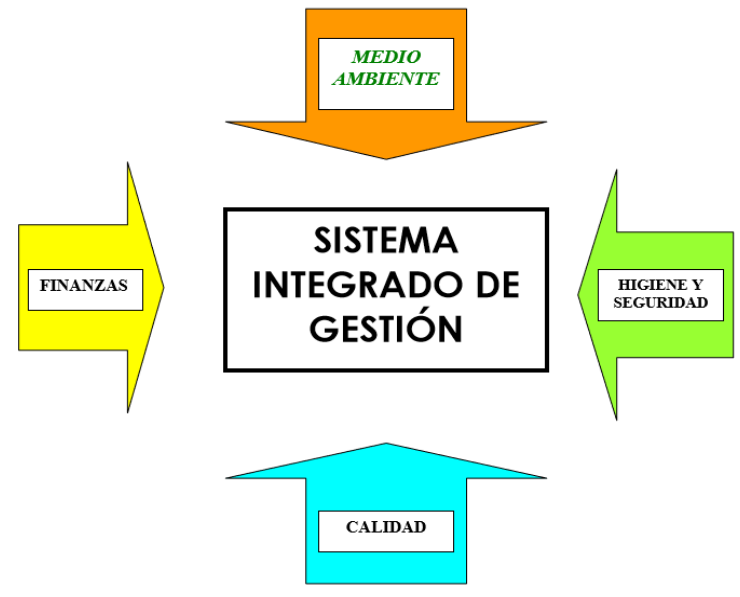

Fuente: (ISO 14001, 2015)

La figura 2 indica que el Sistema Integrado de Gestión, es una herramienta muy valiosa para los proveedores, porque es un sustento trascendental en las organizaciones adoptar la Normativa ISO, para el desarrollo de sus productos en el contraste de amplios datos de mercado de sus sectores, permitiendo así competir eficazmente en los mercados del mundo; debido a que integra el medio ambiente, higiene y seguridad, la calidad, y las finanzas. 
Figura 3. Proceso de Gestión Ambiental

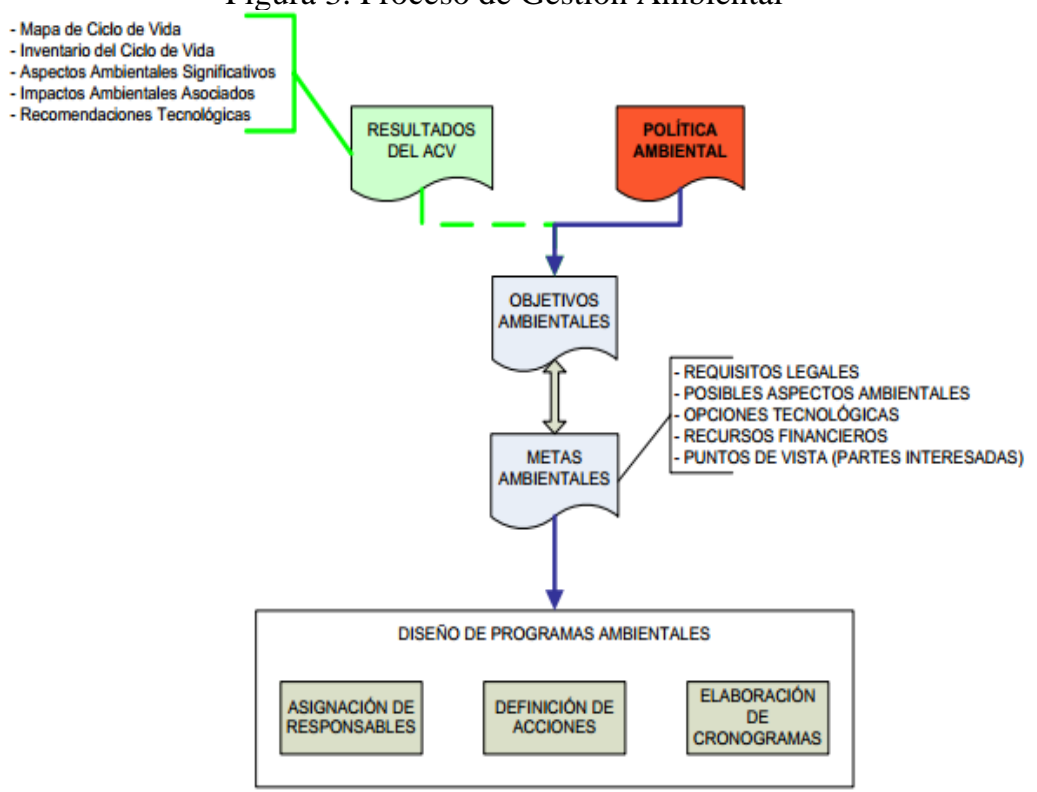

Fuente: (ISO 14001, 2015)

La auditoría de un sistema de gestión ambiental tiene la intención determinar la eficacia de este. La eficacia se entiende como la capacidad de un sistema para asegurar el logro de los fines de la gestión ambiental considerada.

Lo primero que se determina para elaborar un sistema de Gestión Ambiental como lo muestra la Figura 3, es establecer los resultados del Análisis del Ciclo de Vida, la estructura organizativa, las actividades de planificación, responsabilidad, procesos, procedimientos, recursos que conllevan realizar esta gestión ambiental, y mantener una política ambiental en la organización que se lidera, ya sea esta organización pública y privada.

En las instituciones públicas están normadas por la ley No. 1178 de Administración y Control Gubernamental, la que se enmarca en la protección y conservación del medio ambiente y los recursos naturales en el marco del desarrollo sostenible o sustentable.

Las políticas ambientales son aquellas que las determina la organización, de acuerdo a los principios y normas establecidas en su desempeño ambiental que proviene un marco legal, con el fin de que le permita cumplir sus objetivos y metas. Los objetivos ambientales son las metas generales que una institución tiene para perfeccionar su actuación ambiental, deben estar reflejados en la política ambiental; estos deben ser específicos, reales, alcanzables, cuantificables y coherentes con la legislación ambiental.

Las metas ambientales son aquellas que miden el logro de un objetivo, a través de requisitos legales, posibles aspectos ambientales, opciones tecnológicas, recursos financieros, punto de vista de partes interesadas, se debe tener claro que las metas no son lo mismo que los objetivos pero se encuentran 
estrechamente relacionados, ya que un objetivo puede tener varias metas; las metas deben ser medibles y cuantificables al igual que los objetivos.

Adoptar las Normas ISO en las organizaciones garantiza el ahorro del costo a través de la reducción de residuos y un uso más eficiente de los recursos naturales tales como la electricidad, el agua y el gas; al igual que adoptar la normativa brinda el respaldo de no estar es situaciones de posibles multas y penas futuras por incumplimientos de la legislación ambiental vigente.

En síntesis, la auditoría ambiental favorece a la protección del medio ambiente, porque norma el cumplimiento de las leyes, así como políticas y estándares internos de cada empresa, y es la que evalúa los procesos gestión ambiental con la finalidad en disminuir los riesgos ambientales que provocan el mal uso de las políticas de buenas prácticas de manufactura (BPM), adicional contribuye a realizar las mejoras continuas necesarias para que el proceso se desarrolle de manera óptima hasta lograr obtener la eficiencia en el proceso.

(Palencia ,Huertas, Brito, 2018) mencionan que en la AA se pone a prueba los sistemas de control ambiental establecidos por las ISO 14001:2015; a través de la cual se evalúa y se hace control de la información, políticas y procesos que se establece en esta norma.

La ISO 14001 es un grupo de normas internacionales utilizadas en la Gestión de Sistemas Ambientales son necesarias para medir la actuación que se realizan de acuerdo con las resoluciones y criterios aceptadas internacionalmente, esta norma especifica todo los requisitos y requerimientos que se deben cumplir en el Sistema de Gestión Ambiental.

\subsection{PROCESO DE AUDITORÍA AMBIENTAL}

La AA proporciona una estima objetiva en torno a los elementos de un procedimiento que eficiente y adecuados son para amparar el medio ambiente. El procedimiento AA radica en comprobar y valorar apropiadamente prácticas de las cantidades tomadas por las empresas auditadas para aminorar el riesgo de corrupción ambiental originada por la puesta en marcha de actividades que representan peligros constantes para el medio ambiente.

Existen tres etapas de generales que se detallan a continuación en la Tabla 2.

Tabla 2 Etapas de auditoría ambiental

\begin{tabular}{|l|l|}
\hline ETAPAS & CONCEPTO \\
\hline $\begin{array}{l}\text { PRE } \\
\text { AUDITORÍA }\end{array}$ & Esta es la etapa inicial donde se planea la aplicación de la auditoría en todas sus fases. \\
\hline AUDITORÍA & $\begin{array}{l}\text { En segundo lugar tenemos la etapa en la que se asegura la ejecución del procedimiento } \\
\text { según lo planificado con anterioridad. }\end{array}$ \\
\hline $\begin{array}{l}\text { POST } \\
\text { AUDITORÍA }\end{array}$ & $\begin{array}{l}\text { Finalmente en esta parte se realizan los compromisos adquiridos por la entidad auditada y } \\
\text { la posterior finalización de las actividades de auditoría. }\end{array}$ \\
\hline
\end{tabular}

Fuente: Elaboración propia a partir de (Arévalo Correa, S. R., 2014) 


\subsection{IMPACTO AMBIENTAL.}

(Díaz y Escárcega , 2009), consideró que el impacto ambiental no implica la negatividad, dado que puede ser tanto positivo como negativo, pero se considera como la alteración al medio ambiente en sus componentes provocados por una acción humana.

El impacto ambiental es la conmoción del medio ambiente, provocada de forma directa o indirecta por un proyecto o trabajo en relación con un área en específica, en pocas palabras el impacto ambiental es el cambio causado por el hombre o de la naturaleza.

\subsection{RESPONSABILIDAD SOCIAL}

Es importante entender que la empresa de hoy es diferente porque, entre otros motivos, estamos en un siglo joven donde la realidad se parece poco a la de hace algunos años ya que vivimos en un mundo que se mueve de manera vertiginosa. Otro aspecto importante para considerar es que la tecnología ha accedido hasta los rincones más recónditos de las personas y las empresas, en tal sentido la revolución digital, el internet, los sistemas de comunicación y las redes sociales han venido para quedarse y ahora el poder lo ostenta el individuo (consumidor, cliente, empleado o votante, entre otros).

Lo anterior ha traído como consecuencia que el papel de las empresas en la sociedad haya cambiado y los líderes empresariales representen "la cara y el alma" de la organización frente a los diversos grupos de interés. En este contexto nace la Responsabilidad Social Empresarial (RSE) como un conjunto de prácticas de la organización que deben proporcionar un beneficio tanto para la organización como para la sociedad.

La RSE por tanto "es un modo de conducir negocios por parte de las empresas, en la cual se busca que los mismos sean económicamente rentables, apegados a la ley, éticamente correctos y socialmente solidarios" (De la Cruz, 2012; Carroll, 1983, 1999), por tanto el tema ha pasado de ser socializado únicamente en las aulas de las universidades para convertirse en práctica diaria de las empresas del país, impactando positivamente en la sociedad.

El país ha presentado en las últimas décadas un interés por el crecimiento, desarrollo, del ecosistema y el medio ambiente, contribuyendo con la responsabilidad social en el cumplimiento de la preservación del mismo, y la transformación de la matriz productiva. Por tal motivo, y atendiendo a estas necesidades, los docentes investigadores de las Universidades Laica Vicente Rocafuerte, De Guayaquil y Península de Santa Elena, se ven comprometidos a trabajar con responsabilidad social de país y se permiten desarrollar una investigación de Gestión Ambiental, basado en la Norma ISO 14001:2015, en el sector empresarial hotelero de la ciudad de Guayaquil. 
Sin embargo, (Plaza Úbeda, De Burgos-Jiménez, 2011), sostienen que la situación económica actual obliga a las empresas a mejorar su rendimiento financiero pero a la vez hace que sea necesario mejorar también las prácticas de gestión medio ambiental en las empresas.

La Auditoría Ambiental solicitada en el desarrollo del control interno, permite identificar las áreas y los procesos que afectan a los recursos naturales del medio ambiente, esta herramienta permite que se cumplan los reglamentos, normativas, leyes y procedimientos vigentes bajo la norma ISO 14001 en la empresa u organizaciones, es fundamental para responsabilidad social y empresarial.

\section{MÉTODOS Y HERRAMIENTAS}

\subsection{CONSIDERACIONES GENERALES}

Los aspectos metodológicos de la investigación permitirán dar respuestas a interrogantes que tienen que ver con lo que se quiere estudiar, los alcances, el por qué, los fundamentos teóricos y los aspectos que se pretende probar. En otras palabras, es el cómo se va a llevar a cabo el estudio y responde al problema planteado en los objetivos específicos de la investigación. Vale mencionar que para toda investigación es importante tener una metodología.

Los elementos metodológicos de la investigación están conformados por métodos, registros y técnicas, que ayudaran a obtener la información que se necesita para la investigación propuesta. En este orden de ideas, el estudio se dividió en dos fases, la primera de análisis documental, planificación y elaboración de instrumentos y la cual se presenta en este documento y sienta las bases de la fase siguiente donde se realizará el trabajo de campo con su respectivo análisis de datos y elaboración del manual.

\subsection{TIPO DE INVESTIGACIÓN}

El estudio se caracterizó por ser una investigación descriptiva con énfasis en aplicar el análisis de datos con los cuales se presentan los hechos o situaciones que, dada su semejanza, se hace necesario describir sistemáticamente a fin de evitar posibles errores en su manejo.

\subsection{FUENTES DE RECOLECCIÓN DE DATOS}

En la investigación documental, el análisis de los documentos es una técnica que suministra información muy importante en cualquiera de las etapas del proceso investigativo.

Las fuentes primarias consistieron en los documentos oficiales, Normas Internacionales, además de libros de texto. Como método de análisis de los documentos se utilizaron el análisis interpretativo, el cual se define como "el conjunto de operaciones lógicas que se efectúan para interpretar el contenido del informe desde la perspectiva de la investigación" y el análisis formalizado definido como "el conjunto de 
métodos cuantitativos para interpretar el contenido del documento, con la finalidad de encontrar marcas y propiedades susceptibles de calcular” (GONZÁLEZ, A. - GALLARDO, T., 2007 pág. 78). Para el análisis interpretativo los investigadores se plantearon el objetivo de estudio y revelaron la esencia de los documentos, su información patente y latente, de acuerdo al propósito de la investigación.

\section{RESULTADOS}

La ciudad de Guayaquil es el puerto más importante del país, y a través de él se generan divisas que son ingresos para el Presupuesto General del Estado, conscientes de aportar con el ambiente y el ecosistema, se dinamiza el trabajo investigativo, por medio de la estandarización de Políticas y Manuales de Gestión Ambiental, que regulan al sector empresarial Hotelero, siendo este uno de los pilares fundamentales que sostienen la economía turística y la Matriz Productiva del Buen Vivir.

Con la ejecución del presente proyecto se prevé contribuir al fortalecimiento de la política ambiental Nacional y al desarrollo del Plan Nacional del Buen Vivir, directamente con el objetivo estratégico 7.9 "Promover patrones de consumo conscientes, sostenibles y eficientes con criterio de suficiencia dentro de los límites del planeta."

Como primer paso, dentro del proceso investigativo se identificarán las políticas ambientales presentes en el sector empresarial hotelero, mediante el análisis del marco legal vigente en el país y el grado de cumplimiento de la normativa ambiental, para esto se visitarán los principales hoteles de la ciudad que son parte de la muestra de la investigación.

Se realizará el proceso diagnóstico de la auditoría ambiental en el sector empresarial hotelero de la ciudad, una vez obtenido los resultados que serán evidenciados a través de encuestas, entrevistas y grupos focales se obtendrán las tabulaciones y los resultados, que van a permitir realizar el diseño del Manual de Buenas Prácticas de Manufactura y el de Gestión de Auditoria Ambiental.

Los actores que formaran parte del proyecto son el GAD Guayaquil (Gobierno Autónomo Descentralizado), Asociación Empresaria Hotelera Gastronómica de la ciudad de la Plata y su zona de Influencia así como la Asociación de Hoteleros del Guayas.

\section{CONCLUSIONES}

- Las auditorías ambientales son de gran importancia en las organizaciones, las mismas que deben trabajar con un alto grado de ética y responsabilidad ambiental en el medio en el que desarrollan sus actividades económicas. 
- A través de la auditoría ambiental las organizaciones ganan reputación, ahorran costos, existe la mejora continua en el proceso, se ven motivadas al cumplimiento de leyes y manejan sistemas integrados.

- El cambio globalizado del mundo y el cuidado por el ecosistema, nos lleva a tomar responsabilidad ética y moral en los cambios transformadores e innovadores de la ciencia y la tecnología, evocamos a unir fuerzas, para todos ser parte de esta visión integradora y social que conlleve a mejorar el país a través de los recursos renovables que posee.

- Es importante que en el país exista más auditores ambientales con sentido ético y de responsabilidad que posean conocimiento en la Normativa ISO, ya que estas contribuyen a mejorar el comportamiento ambiental y las oportunidades de beneficio económico.

- El Ecuador a través de las normativas busca establecer procesos que evalúen el impacto ambiental que generan las diferentes actividades económicas que realizan las organizaciones, con el objetivo de crear conciencia medioambiental en los administradores y los miembros de la misma.

- La responsabilidad social es un tema de todos, puesto que de una u otra forma somos parte de una actividad económica la misma que puede generar un impacto significativo al medio ambiente. 


\section{REFERENCIAS BIBLIOGRÁFICAS}

Antúnez Sánchez. 2015. La auditoría ambiental, la empresa amigable con el ambiente y el desarollo sostenible. s.l. : Revista de Ciencias Sociales de la Universidad, 2015.

Arévalo Correa, S. R. 2014. La auditoría medio ambiental en la determiancioón de la responsabilidad social en las empresa mineras Gold Fields La Cima S.A. . 2014.

ATRISTAIN - ÁLVAREZ. 1999. LA RESPONSABILIDAD DE LA CONTABILIDAD CON EL MEDIO AMBIENTE. MÉXICO : s.n., 1999. pág. 117.

Cuevas Barros, C., \& Cuevas Casas, C. 2013. La Auditoría Ambiental (AA) una mirada pedagógica. s.l.

: Universidad de Ciencias Pedagógicas "Hector Alfredo Pineda Zaldivar”., 2013.

Díaz y Escárcega . 2009. Desarrollo sustentable - oportunidad para la vida. Mexico : Editorial McGrawHill. , 2009.

Franklin, E. B. 2015. Auditoría Administrativa. Evaluación . México : Pearson Education., 2015.

GABIÑO, MARCELO. 1999. AUDITORÍAS AMBIENTALES. DEPARTAMENTO HIDRÁULICA, UNIVERSIDAD NACIONAL DE LA PLATA. LA PLATA : s.n., 1999.

GONZÁLEZ, A. - GALLARDO, T. 2007. INVESTIGACIÓN EDUCATIVA. 2007.

ISO 14001, NORMA INTERNACIONAL. 2015. NORMA INTERNACIONAL ISO 14001. 2015.

Mejía, H. A. 2015. Responsabilidad ambiental administrativa, un análisis de la aplicación de los principios del Derecho Administrativo . 2015.

Palencia „Huertas, Brito. 2018. Importancia de la implementación de la auditoría ambiental en la prespectiva de la gestion ambiental de las empresas. 2018.

PEQUERO - MEDINA - CAMPOS. 2008. CONTABILIDAD Y AUDITORÍA PARA EL DESARROLLO SUSTENTABLE, EL RECONOCIMIENTO DEL INSTITUTO MEXICANO DE CONTADORES PÚBLICOS. 2008.

Plaza Úbeda, De Burgos-Jiménez. 2011. Grupos de interés, gestión ambiental y resultado empresarial: una propuesta. 2011.

Reyes y Moreno. 2017. El control ambiental a través de la auditoría como instrumento empresarial. 2017.

Sotomayor, A. A. 2008. Contabilidad Administrativa. México : Hill Interamericana Editores S.A., 2008. 